\title{
Expression of ARs in triple negative breast cancer tumors: a potential prognostic factor?
}

\author{
This article was published in the following Dove Press journal: \\ OncoTargets and Therapy \\ 23 July 2015 \\ Number of times this article has been viewed
}

\begin{abstract}
Aris Giannos'
Martin Filipits ${ }^{2}$

Flora Zagouri ${ }^{2,3}$

Anita Brandstetter ${ }^{2}$

Alexandra Tsigginou'

Maria Sotiropoulou ${ }^{4}$

Irene Papaspyrou ${ }^{4}$

Theodoros N Sergentanis ${ }^{5}$

Theodora Psaltopoulou ${ }^{5}$

Alexandros Rodolakis'

Aris Antsaklis'

Meletios-Athanasios

Dimopoulos ${ }^{2}$

Constantine Dimitrakakis'

'Department of Obstetrics and Gynaecology, Alexandra General

Hospital, Medical School, University

of Athens, Athens, Greece; ${ }^{2}$ Institute

of Cancer Research, Department of

Medicine I, Comprehensive Cancer

Center, Medical University of Vienna,

Vienna, Austria; ${ }^{3}$ Department of

Clinical Therapeutics, Alexandra

General Hospital, Medical School,

University of Athens, Athens,

Greece; ${ }^{4}$ Department of Pathology,

Alexandra General Hospital, Athens,

Greece; ${ }^{5}$ Department of Hygiene,

Epidemiology and Medical Statistics,

Medical School, University of Athens,

Athens, Greece
\end{abstract}

Correspondence: Flora Zagouri

Department of Clinical Therapeutics,

Alexandra General Hospital, Medical

School, University of Athens, Vas Sofias

Ave and Lourou Street, Athens II52I,

Greece

$\mathrm{Tel}+30213216254 \mid$

Fax +3021321625II

Email florazagouri@yahoo.co.uk
Background/aim: In light of the controversial published literature, this study aims to examine the potential prognostic role of AR immunohistochemical expression in triple negative breast cancer (TNBC).

Patients and methods: Ninety patients with TNBC were included in this study; the associations between AR expression (Allred score), clinicopathological variables (stage, grade, histological subtype, tumor size, nodal status, age at diagnosis, Ki67 expression, and p53 expression), and overall survival were evaluated.

Results: AR expression was not associated with stage, grade, histological subtype, tumor size, nodal status, age at diagnosis, Ki67 expression, and p53 expression. AR immunopositivity was not associated with overall survival either at the univariate or at the multivariate Cox regression analysis (multivariate hazard ratio $=0.66,95 \%$ confidence interval: $0.26-1.70, P=0.393$ ).

Conclusion: AR expression does not seem to play a prognostic role in TNBC.

Keywords: biomarkers, prognosis, AR, triple negative breast cancer

\section{Introduction}

Triple negative breast cancer (TNBC) is an entity that is characterized by ER, PR, and HER2 negativity. TNBC is characterized by poor prognosis, ${ }^{1,2}$ as well as more frequent metastases in lungs and brain;, ${ }^{3,4}$ chemotherapy represents the available effective modality of systemic therapy for this type of breast cancer.

Immunohistochemical studies have shown that ARs are expressed in approximately $70 \%-90 \%$ of invasive breast cancer ${ }^{5-8}$ nevertheless, their role in the pathogenesis of breast cancer remains to be fully elucidated, together with the potential biochemical mechanisms. It has been suggested that androgens may mediate a multifaceted role; they may induce a growth-inhibitory response in breast cancer cell lines expressing ER, PR, and AR, such as T-47-D and ZR-75-1, whereas growth-inducing effects have been noted in the $\mathrm{ER}(+)$ and $\mathrm{PR}(+) \mathrm{MCF}-7$ cells. ${ }^{9}$ On the other hand, regarding breast cancer cell lines that do not express ER and PR, but are still AR positive, androgens may exhibit a proliferative effect; therefore interactions with cofactors and co-repressors have been suggested as meaningful for the net effects of androgens in breast cancer. ${ }^{9}$ Concerning potential cofactors, Garay et al highlighted the interaction in the pathways between EGFR and AR on the level of MAPK pathway, as well as the dependence of AR signaling on $\mathrm{p} 21 .{ }^{10}$

A variety of studies have examined the role of ARs in terms of prognosis of breast cancer, with controversial findings. Kuenen-Boumeester et al showed that AR expression was associated with better disease free survival at the univariate analysis but the association dissipated at the multivariate approach; ${ }^{11}$ on the other hand, the Nurses Health Study suggested a non-significant association between AR status and breast

submit your manuscript $\mid$ www.dovepress.con 
cancer death. ${ }^{5}$ AR positivity has been found correlated with negative lymph node metastasis, ${ }^{12}$ smaller tumor size, ${ }^{6,12}$ low histological grade, ${ }^{6,12}$ no elevated preoperative serum cancer antigen 15-3 levels, ${ }^{6}$ and p53-negativity. ${ }^{12}$

The controversy is also apparent among the studies examining AR expression especially in TNBC. Mrklic et al suggested that AR expression correlated inversely with higher clinical stage, histological grade mitotic score and proliferation index measured by $\mathrm{Ki}-67$, but not with disease free survival or overall survival. ${ }^{13}$ On the contrary, the nested analysis within the Nurses Health Study suggested that women with AR-positive TNBC had a sizable and statistically significant (by $83 \%$ ) increase in overall mortality compared with those with AR-negative tumors. ${ }^{5}$

In light of the controversial results, this study aims to examine the potential prognostic role of AR immunohistochemical expression in TNBC. This question seems of special importance, given that a prognostic role for ARs would imply an opportunity for a targeted treatment in this group of breast cancers, whose treatment options are limited.

\section{Patients and methods}

\section{Study sample}

Patients were recruited during a 9-year period (2002-2010) in a tertiary reference center (Department of Clinical and Obstetrics, Alexandra General Hospital). Ninety consecutive cases of Caucasian women, with TNBC, were identified through a detailed database; a centralized histological review of all cases and controls has been performed for the ascertainment of the condition. Information on patients' age, stage, grade, tumor size, nodal status, and histological subtype was obtained from patients' charts; two independent pathologists evaluated the histopathological variables.

Written informed consent was obtained from all participants in this study. This study conforms with the Helsinki Declaration and has been approved by the local Institutional Review Board.

\section{Immunohistochemistry}

To ensure the triple negative nature of the breast cancer cases, PgR (636, Dako Denmark A/S, Glostrup, Denmark), ER (ID5, Dako) and HER2 (CB11, Novocastra ${ }^{\text {TM }}$ ) antibodies were used. Sections (thickness: $4 \mu \mathrm{m}$ ) were cut from formalinfixed paraffin embedded tissues. The breast carcinoma tissue sections were deparaffinized in xylene and hydrated in graded ethanol solutions; thereafter, they were pretreated to enhance antigen retrieval Primary antibodies against PgR, ER, and HER2 were used in the EnVision + System-HRP
(DAB) DakoCytomation (Dako Denmark A/S). Immunohistochemistry was conducted following the manufacturer's instructions.

AR immunohistochemistry was performed at the Institute of Cancer Research, Medical University of Vienna using a standard protocol. Specifically, tissue sections were deparaffinized and rehydrated. Slides were incubated in $0.3 \%$ $\mathrm{H}_{2} \mathrm{O}_{2}$ for 10 minutes to reduce nonspecific background staining. Subsequently, specimens were heated for 10 minutes in $1 \mathrm{mM}$ ethylenediaminetetraacetic acid (EDTA) buffer ( $\mathrm{pH}$ 8.0) in a pressure cooker for epitope retrieval. Specimens were incubated with Ultra V Block (UltraVision LP detection system; Lab Vision Corporation, Fremont, CA, USA) at room temperature for 5 minutes to block background staining, incubation for 60 minutes at room temperature with a mouse monoclonal antibody specific for AR (AR441, Dako) followed. To detect antibody binding, the UltraVision LP detection system was used, following the manufacturer's instructions. Color development with 3-3'-diaminobenzidine was performed and counterstaining with hematoxylin was conducted. Breast cancer sections known to express AR were used as positive controls.

The examination of AR expression was assessed in a blinded manner regarding the patients' clinical data. At least 100 tumor cells were evaluated for each patient. Immunostaining was quantified on the basis of nuclear staining intensity as well as percentage of AR-positive tumor cells using the Allred score. Figure 1 portrays a case with high AR expression.

\section{Statistical methods}

For the evaluation of the association between overall survival and expression of ARs, a standard two-step approach was followed: univariate and multivariate survival analysis; univariate and multivariate Cox regression analysis was undertaken, respectively. An alternative approach, with the AR Allred score treated as a continuous variable, was also followed so as to explore the reproducibility of any findings.

Apart from the overall analysis a subgroup analysis in the most prevalent histological subtype, namely ductal TNBC, was performed. Statistical analysis was performed using STATA/SE 13 statistical software (StataCorp LP, College Station, TX, USA).

\section{Results}

Descriptive statistics of the study sample are presented in Table 1, by AR expression status. AR expression was not associated with stage ( $P=0.444)$, grade $(P=0.696)$, histological 


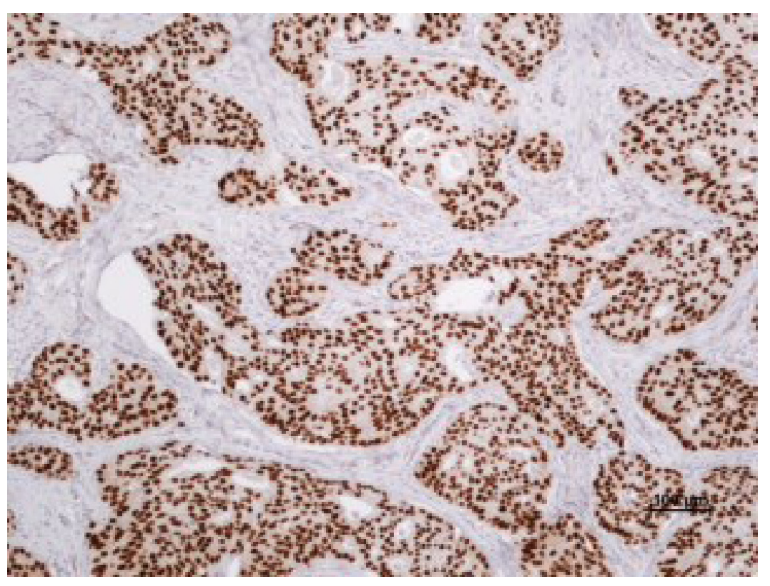

Figure I Examples of AR immunostaining.

Notes: TNBC specimen with high AR expression is shown. Bar $=100 \mu \mathrm{m}$. Abbreviation: TNBC, triple negative breast cancer.

subtype $(P>0.999)$, size of tumor $(P=0.087)$, nodal status $(P=0.080)$, age at diagnosis $(P=0.105), \mathrm{Ki67}$ expression $(P=0.698)$, and 553 expression $(P=0.735)$. Anthracycline and taxane-based chemotherapy was received in $48.9 \%$ of

Table I Descriptive statistics of the study sample

\begin{tabular}{|c|c|c|c|}
\hline \multirow[t]{2}{*}{ Categorical variables } & \multirow{2}{*}{$\begin{array}{l}\text { AR-negative } \\
\text { cases } \\
\mathbf{N}(\%)\end{array}$} & \multirow{2}{*}{$\begin{array}{l}\text { AR-positive } \\
\text { cases } \\
\mathbf{N}(\%)\end{array}$} & \multirow[t]{2}{*}{$P$-value } \\
\hline & & & \\
\hline Stage & & & $0.444^{\mathrm{CT}}$ \\
\hline 1 & $8(17.8)$ & $9(20.0)$ & \\
\hline ॥ & $27(60.0)$ & $20(44.4)$ & \\
\hline III & $10(22.2)$ & $16(35.6)$ & \\
\hline Grade & & & $0.696^{\mathrm{CT}}$ \\
\hline 1 & $2(4.9)$ & $0(0.0)$ & \\
\hline 2 & $3(7.3)$ & $9(20.9)$ & \\
\hline 3 & $36(87.8)$ & $34(79.1)$ & \\
\hline Histological subtype & & & $>0.999^{F}$ \\
\hline Ductal & $33(73.3)$ & $34(75.6)$ & \\
\hline Lobular & $6(6.7)$ & $3(6.7)$ & \\
\hline Other & $9(20.0)$ & $8(17.8)$ & \\
\hline Tumor size & & & $0.087^{\mathrm{CT}}$ \\
\hline TI & $9(20.0)$ & I8 (40.0) & \\
\hline T2 & $29(64.4)$ & $23(5 \mathrm{I} . \mathrm{I})$ & \\
\hline $\mathrm{T} 3$ & $5(11.1)$ & $2(4.4)$ & \\
\hline $\mathrm{T} 4$ & $2(4.4)$ & $2(4.4)$ & \\
\hline Nodal status & & & $0.080^{\mathrm{CT}}$ \\
\hline No & $31(68.9)$ & $22(48.9)$ & \\
\hline $\mathrm{NI}$ & $4(8.9)$ & $7(15.6)$ & \\
\hline N2 & $4(8.9)$ & $5(11.1)$ & \\
\hline N3 & $6(13.3)$ & II (24.4) & \\
\hline Continuous variables & Mean \pm SD & Mean \pm SD & $P$-value \\
\hline Age at diagnosis (years) & $53.9 \pm \mid 5.1$ & $59.4 \pm 12.7$ & $0.105^{\mathrm{M}}$ \\
\hline Ki67 expression (\%) & $34.9 \pm 21.2$ & $38.1 \pm 27.3$ & $0.698^{\mathrm{M}}$ \\
\hline P53 expression (\%) & $40.8 \pm 40.2$ & $36.6 \pm 46.0$ & $0.735^{\mathrm{M}}$ \\
\hline
\end{tabular}

Notes: ${ }^{C T} P$-value derived from chi-square test for trend; ${ }^{F} P$-value derived from Fisher's exact test; ${ }^{M} P$-value derived from Mann-Whitney-Wilcoxon test for independent samples.

Abbreviation: SD, standard deviation. patients, $24.4 \%$ received anthracycline-based chemotherapy, $12.2 \%$ taxane-based chemotherapy, 6.7\% CMF regimen, whereas $7.8 \%$ did not receive chemotherapy. In our series no cases presented with metastases; therefore, a relevant analysis is not presented.

The subgroup analysis in ductal TNBC $(n=67)$ replicated the lack of associations; AR was not associated with stage ( $P=0.391)$, grade $(P=0.092)$, tumor size $(P=0.195)$, nodal status $(P=0.119)$, age at diagnosis $(P=0.091)$, Ki67 expression $(P=0.393)$, and $\mathrm{p} 53$ expression $(P=0.853)$.

The median follow-up (defined as the median follow-up among those patients who did not die) was 87 months; the 5 -year overall survival was $84.0 \%$. At the univariate survival analysis, AR expression was not associated with overall survival either when it was treated as a binary variable (univariate hazard ratio [HR] $=1.13,95 \%$ confidence interval [CI]: $0.47-2.69, P=0.784$ for AR positivity versus negativity) or as a continuous variable (univariate $\mathrm{HR}=0.99$, 95\% CI: $0.85-1.14, P=0.871$ for 1-point increase in the Allred score) in the total TNBC sample. Figure 2 presents the Kaplan-Meier overall survival curves for AR-positive and AR-negative cases.

The results of the multivariate Cox regression analysis are presented in Table 2. Apart from an association between poorer survival and more advanced stage (multivariate $\mathrm{HR}=2.82,95 \%$ CI: $1.33-5.99, P=0.007$ for one level increase), no associations implicating grade, age at diagnosis, and AR immunopositivity (multivariate $\mathrm{HR}=0.66,95 \%$ CI: $0.26-1.70, P=0.393$, versus AR negativity) were detected. An alternative model with AR Allred score treated as continuous variable replicated the lack of association with overall survival (multivariate HR $=0.89,95 \%$ CI: $0.75-1.04$, $P=0.136$ for 1-point increase in the Allred score).

The subgroup analysis on ductal carcinomas similarly yielded non-significant associations in both approaches to AR expression (multivariate $\mathrm{HR}=0.73,95 \%$ CI: $0.24-2.28$, $P=0.593$ for the binary approach and multivariate $\mathrm{HR}=0.91$, 95\% CI: $0.76-1.09, P=0.289$ for the continuous approach).

\section{Discussion}

The main findings of this study pertain to the lack of a significant prognostic effect mediated by AR immunohistochemical expression. AR positivity was not associated either with the compendium of clinicopathological findings (grade, stage, histological type, tumor size, nodal status, Ki67 expression, and $\mathrm{p} 53$ expression) or with overall survival; the null associations were replicated in the subgroup of ductal carcinomas. 


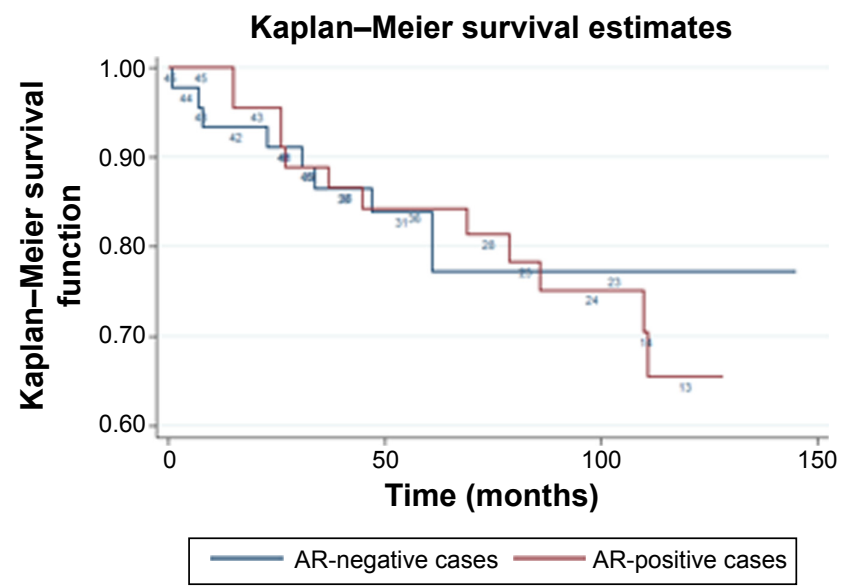

Figure 2 Kaplan-Meier overall survival curves for AR-positive and AR-negative cases.

The published literature has yielded extremely controversial findings regarding the potential prognostic role of AR expression in TNBC. Similarly to our findings, Mrklic et al supported that AR expression was not associated with disease free survival or overall survival; nevertheless, their study pointed to an indirect prognostic role, as AR expression correlated inversely with higher mitotic score, clinical stage, histological grade, and Ki-67 proliferation index. ${ }^{13}$ A similar pattern was noted in the study by Rakha et al where the multivariate analysis did not point to any independent prognostic effect mediated by AR expression in TNBC, despite its associations with grade, development of recurrences, and distant metastases. ${ }^{14}$ In accordance with our findings, and adopting a more sophisticated methodology, namely reverse-phase protein arrays to measure AR levels, Gonzalez-Angulo et al supported that AR expression did not correlate with either overall or relapse-free survival in TNBC despite a borderline trend toward higher AR expression among cases with PIK3CA mutations. ${ }^{15}$

In contrast with the null association detected in our sample, Luo et al supported a multifaceted favorable prognostic role of AR, as its expression was associated with tumor grade, nodal status, disease free survival, and overall survival in their series of TNBC.${ }^{16}$ Enlarging the perspective,

Table 2 Results of multivariate Cox regression analysis for overall survival

\begin{tabular}{llll}
\hline Variables & $\begin{array}{l}\text { Category or } \\
\text { increment }\end{array}$ & HR $(\mathbf{9 5} \% \mathbf{C l})$ & $\boldsymbol{P}$-value \\
\hline AR positivity & Yes versus no & $0.66(0.26-1.70)$ & 0.393 \\
Stage & One level increase & $\mathbf{2 . 8 2 ( 1 . 3 3 - 5 . 9 9 )}$ & 0.007 \\
Grade & One level increase & $0.60(0.24-1.5 \mathrm{I})$ & 0.282 \\
Age at diagnosis & One year increase & $\mathrm{I} .02(0.99-1.06)$ & $0.24 \mathrm{I}$ \\
\hline
\end{tabular}

Note: Bold values denote a statistically significant association.

Abbreviations: $\mathrm{HR}$, hazard ratio; $\mathrm{Cl}$, confidence interval.
Agoff et al examined ER-negative breast cancer cases, highlighting an association of AR expression with better disease free survival, lower grade, and smaller size. ${ }^{17}$ On the other edge of the spectrum, AR expression was portrayed as an unfavorable prognostic factor in the nested analysis within the Nurses Health Study. ${ }^{5}$

The AR positivity rate demonstrated in our TNBC series (equal to 50\%) is compatible with the literature; Luo et al showed 27.7\% positive immunostaining for AR, ${ }^{16}$ Mrklic et al 32.5\%, ${ }^{13}$ Park et al 35\%, ${ }^{6}$ Ogawa et al 43\%, ${ }^{12}$ and Qi et al $53.2 \% .^{7}$ Nevertheless, occasionally lower frequencies of AR expression among TNBC have been reported, with Rakha et al presenting 13\% AR immunopositivity rate in their series $^{14}$ and Gonzalez-Angulo et al reporting $16.5 \%$ high AR levels via reverse-phase protein arrays. ${ }^{15}$

Targeting AR with androgen-based hormonal therapy would represent an attractive treatment option for TNBC cases; nevertheless, our present, null results do not seem to justify AR-targeting therapy. The mechanisms underlying the present null findings remain elusive for the time being; ARs seem to exert pluripotent effects in breast cancer and interactions with cofactors and co-repressors ${ }^{9}$ may limit the net effects of their expression in TNBC; further subgrouping by the expression of potential cofactors may yield interesting results in future studies.

Despite the originality of this study, some limitations should be discussed and addressed. The quantification of AR expression was based on immunohistochemistry; more elaborate techniques, such as Western blot, would seem necessary for further validation of the present findings. At any case, the results of the alternative analysis treating AR expression as a continuous variable (Allred score) replicated the findings of the main analysis. Moreover, disease free survival was not available in our setting; therefore, the reproducibility of the overall survival-related findings upon disease free survival could not be examined. Furthermore, information regarding additional molecular indices associated with AR expression, such as PIK3CA mutations, was not available in our study. ${ }^{15}$ Finally, our results should be further validated in larger studies, as our small sample size may have limited the statistical power in our analysis.

In conclusion, $\mathrm{AR}$ expression did not seem to exert a prognostic effect in our TNBC series, either in terms of clinicopathological variables or overall survival. Future studies adopting more elaborate techniques seem desirable to further validate the present findings and uncover the potential underlying mechanisms, cofactors, and molecular pathways. 


\section{Disclosure}

The authors have no conflicts of interest to disclose.

\section{References}

1. van de Rijn M, Perou CM, Tibshirani R, et al. Expression of cytokeratins 17 and 5 identifies a group of breast carcinomas with poor clinical outcome. Am J Pathol. 2002;161(6):1991-1996.

2. Rouzier R, Perou CM, Symmans WF, et al. Breast cancer molecular subtypes respond differently to preoperative chemotherapy. Clin Cancer Res. 2005;11(16):5678-5685.

3. Hicks DG, Short SM, Prescott NL, et al. Breast cancers with brain metastases are more likely to be estrogen receptor negative, express the basal cytokeratin CK5/6, and overexpress HER2 or EGFR. Am J Surg Pathol. 2006;30(9):1097-1104.

4. Fulford LG, Reis-Filho JS, Ryder K, et al. Basal-like grade III invasive ductal carcinoma of the breast: patterns of metastasis and long-term survival. Breast Cancer Res. 2007;9(1):R4.

5. Hu R, Dawood S, Holmes MD, et al. Androgen receptor expression and breast cancer survival in postmenopausal women. Clin Cancer Res. 2011; 17(7): 1867-1874

6. Park S, Koo J, Park HS, et al. Expression of androgen receptors in primary breast cancer. Ann Oncol. 2010;21(3):488-492.

7. Qi JP, Yang YL, Zhu H, et al. Expression of the androgen receptor and its correlation with molecular subtypes in 980 Chinese breast cancer patients. Breast Cancer (Auckl). 2012;6:1-8.

8. Soreide JA, Lea OA, Varhaug JE, Skarstein A, Kvinnsland S. Androgen receptors in operable breast cancer: relation to other steroid hormone receptors, correlations to prognostic factors and predictive value for effect of adjuvant tamoxifen treatment. Eur J Surg Oncol. 1992;18(2):112-118.
9. Birrell SN, Hall RE, Tilley WD. Role of the androgen receptor in human breast cancer. J Mammary Gland Biol Neoplasia. 1998;3(1):95-103.

10. Garay JP, Karakas B, Abukhdeir AM, et al. The growth response to androgen receptor signaling in ERalpha-negative human breast cells is dependent on p21 and mediated by MAPK activation. Breast Cancer Res. 2012;14(1):R27.

11. Kuenen-Boumeester V, Van der Kwast TH, Claassen CC, et al. The clinical significance of androgen receptors in breast cancer and their relation to histological and cell biological parameters. Eur J Cancer. 1996; 32A(9):1560-1565.

12. Ogawa Y, Hai E, Matsumoto K, et al. Androgen receptor expression in breast cancer: relationship with clinicopathological factors and biomarkers. Int J Clin Oncol. 2008;13(5):431-435.

13. Mrklic I, Pogorelic Z, Capkun V, Tomic S. Expression of androgen receptors in triple negative breast carcinomas. Acta Histochem. 2013; 115(4):344-348.

14. Rakha EA, El-Sayed ME, Green AR, Lee AH, Robertson JF, Ellis IO. Prognostic markers in triple-negative breast cancer. Cancer. 2007; 109(1):25-32.

15. Gonzalez-Angulo AM, Stemke-Hale K, Palla SL, et al. Androgen receptor levels and association with PIK3CA mutations and prognosis in breast cancer. Clin Cancer Res. 2009;15(7):2472-2478.

16. Luo X, Shi YX, Li ZM, Jiang WQ. Expression and clinical significance of androgen receptor in triple negative breast cancer. Chin J Cancer. 2010;29(6):585-590.

17. Agoff SN, Swanson PE, Linden H, Hawes SE, Lawton TJ. Androgen receptor expression in estrogen receptor-negative breast cancer. Immunohistochemical, clinical, and prognostic associations. Am J Clin Pathol. 2003;120(5):725-731.
OncoTargets and Therapy

\section{Publish your work in this journal}

OncoTargets and Therapy is an international, peer-reviewed, open access journal focusing on the pathological basis of all cancers, potential targets for therapy and treatment protocols employed to improve the management of cancer patients. The journal also focuses on the impact of management programs and new therapeutic agents and protocols on

\section{Dovepress}

patient perspectives such as quality of life, adherence and satisfaction. The manuscript management system is completely online and includes a very quick and fair peer-review system, which is all easy to use. Visit http://www.dovepress.com/testimonials.php to read real quotes from published authors. 